Article

\title{
A Complete Design of a Rare Earth Metal-Free Permanent Magnet Generator
}

\author{
Petter Eklund ${ }^{1, *}$, Stefan Sjökvist ${ }^{1}$, Sandra Eriksson ${ }^{1}$ and Mats Leijon ${ }^{1,2}$ \\ ${ }^{1}$ Division of Electricity, Department of Engineering Sciences, Uppsala University, Box 534, \\ Uppsala SE-751 21, Sweden; E-Mails: Stefan.Sjokvist@angstrom.uu.se (S.S.); \\ Sandra.Eriksson@angstrom.uu.se (S.E.); Mats.Leijon@angstrom.uu.se (M.L.) \\ ${ }^{2}$ Faculty of Engineering and the Environment, University of Southampton, \\ Southampton SO17 1BJ, UK \\ * Author to whom correspondence should be addressed; E-Mail: Petter.Eklund@ angstrom.uu.se; \\ Tel.: +46-18-471-58-49; Fax: +46-18-471-58-10.
}

Received: 25 February 2014; in revised form: 2 April 2014 / Accepted: 13 May 2014 /

Published: 16 May 2014

\begin{abstract}
The price of rare-earth metals used in neodymium-iron-boron $(\mathrm{NdFeB})$ permanent magnets (PMs) has fluctuated greatly recently. Replacing the NdFeB PMs with more abundant ferrite PMs will avoid the cost insecurity and insecurity of supply. Ferrite PMs have lower performance than $\mathrm{NdFeB} \mathrm{PMs} \mathrm{and} \mathrm{for} \mathrm{similar} \mathrm{performance} \mathrm{more} \mathrm{PM} \mathrm{material} \mathrm{has} \mathrm{to}$ be used, requiring more support structure. Flux concentration is also necessary, for example, by a spoke-type rotor. In this paper the rotor of a $12 \mathrm{~kW} \mathrm{NdFeB} \mathrm{PM} \mathrm{generator} \mathrm{was} \mathrm{redesigned}$ to use ferrite PMs, reusing the existing stator and experimental setup. Finite element simulations were used to calculate both electromagnetic and mechanical properties of the design. Focus was on mechanical design and feasibility of construction. The result was a design of a ferrite PM rotor to be used with the old stator with some small changes to the generator support structure. The new generator has the same output power at a slightly lower voltage level. It was concluded that it is possible to use the same stator with either a $\mathrm{NdFeB}$ PM rotor or a ferrite PM rotor. A ferrite PM generator might require a larger diameter than a $\mathrm{NdFeB}$ generator to generate the same voltage.
\end{abstract}

Keywords: rare earth metal-free; permanent magnet; synchronous generator 


\section{Introduction}

In the light of increasing prices of Neodymium-Iron-Boron, $\mathrm{NdFeB}$, permanent magnets, PMs, it has become interesting to replace them with weaker but cheaper ferrite PMs [1]. To investigate if such substitution is possible, an experimental generator for wind power, built using NdFeB PMs, was chosen to be redesigned to use ferrite PMs. In the redesign of the generator the stator was not to be changed, but the rotor and support structure could be replaced as needed.

The generator to be redesigned in this paper is part of an experimental setup, and is identical to the generator used in a $12 \mathrm{~kW}$ research wind turbine. The original design is described by Eriksson et al. in $[2,3]$, and achieves the rated power at a speed of $127 \mathrm{rpm}$ and operates at variable speed.

There have been earlier reports on ferrite PM magnetized synchronous generators. However, the more powerful $\mathrm{NdFeB}$ PMs have been much more common in generators since their introduction. There is a great difference in magnetic performance between ferrite and $\mathrm{NdFeB}$. The typical value of the remanence for ferrites is $0.4 \mathrm{~T}$ while it is around $1.3 \mathrm{~T}$ for NdFeB. For the maximum energy product the difference is even larger. For ferrite PM the maximum energy product is typically $32 \mathrm{~kJ} \cdot \mathrm{m}^{-3}$ while it for $\mathrm{NdFeB}$ $\mathrm{PMs}$ is $317 \mathrm{~kJ} \cdot \mathrm{m}^{-3}$. Ferrites are also more sensitive to demagnetization [4].

There are different ways of mounting the ferrite PMs. In 1979, Binns and Kurdali proposed a radial flux design with axially magnetized magnets between flux concentrating flux guides. The design was claimed to be suitable for both ferrite and rare-earth PMs [5]. Another possibility is an axial flux machine proposed by Muljadi et al. in 1999 for use with either ferrite or NdFeB PMs [6]. A design with surface mounted ferrite PMs have also been reported by Jang et al. in 2012 [7].

Most ferrite PM magnetized machines are of a spoke configuration. In a spoke configuration the PMs are tangentially magnetized and placed between pole shoes directing the magnetic flux into the air gap. This allows for flux concentration. Numerous variations have been reported on this basic concept, such as a cable wound machine [8], machines with a rotor with a large overhang [9], and modular designs [10]. The concept have recently been tested experimentally in linear generators for wave power as reported by Ekergård et al. [11].

Previous work of the research group on converting a NdFeB PM generator to using ferrite PMs has been published by Eriksson and Bernhoff, describing the electromagnetic design of a ferrite PM rotor intended to be used interchangeably with a $\mathrm{NdFeB} \mathrm{PM}$ rotor in the same stator [1]. However, no full mechanical design was made in that study, while in this work a full mechanical design have been coupled with the electromagnetic design. This have constrained the design as presented in Section 2.2. In this paper, a complete design for conversion of a generator with premagnetized $\mathrm{NdFeB}$ magnets into a novel design with ferrite magnets is presented.

The new design is currently under construction.

\section{Design Process and Simulations}

\subsection{The Original NdFeB Magnetized Generator}

The original $\mathrm{NdFeB}$ generator has an outer stator diameter of $886 \mathrm{~mm}$, and the stator stack has a nominal length of $222 \mathrm{~mm}$ [2]. A summary of the characteristics of the original design is given in Table 1. 
Table 1. Characteristics at rated power of the original generator.

\begin{tabular}{lc}
\hline Rated power [kW] & 12.0 \\
No load phase voltage at rated speed (rms) [V] & 172 \\
Phase voltage at rated power (rms) [V] & 167 \\
Electrical frequency at rated power [Hz] & 33.9 \\
Rotational speed at rated power [rpm] & 127 \\
Number of poles & 32 \\
Number of slots per pole and phase & $5 / 4$ \\
Stator inner diameter [mm] & 760 \\
Stator outer diameter [mm] & 886 \\
Air gap width [mm] & 10 \\
Stator length [mm] & 222 \\
\hline
\end{tabular}

Two machines have been built according to the $\mathrm{NdFeB}$ magnetized design. Both of these have been tested and operated as parts of experiments. One was installed in a $12 \mathrm{~kW}$ research wind turbine in 2006 and the other is part of a laboratory experimental setup. The latter of these will be rebuilt according to the design presented in this paper. Since the machine is operated at variable speed, grid connection is facilitated through a full converter.

\subsection{Design Requirements}

The requirement to keep the old stator imposes restrictions on the new rotor. The rotor diameter is limited by the required air gap, at least $7 \mathrm{~mm}$, and stator inner diameter, of $760 \mathrm{~mm}$. The number of poles are 32 and the number of slots per pole and phase is fixed at $\frac{5}{4}$.

In the original design a large air gap of $10 \mathrm{~mm}$ was chosen for ease of construction and robustness. Due to the lower performance of the ferrite PMs it will be decreased to $7 \mathrm{~mm}$.

The generator is intended to be used as an experimental prototype and only one will be built. Therefore ease of small series manufacture have been a priority in the design.

Magnet size has been limited by the largest rotor diameter allowed, as well as the required room for fasteners on the end of the of the pole shoes furthest from the air gap. Another requirement, imposed by the single unit series, was to use standard size magnets. Because of concerns about handling of the PMs, due to strong magnetic forces, it was decided that the design should be adapted for inserting the magnets after the rotor was mounted in the stator and this mounting procedure needed to be considered during the whole design process.

\subsection{Simulation Method}

Electromagnetic simulations of the ferrite PM generator was performed using a two dimensional cross section of the geometry, perpendicular to the axis of rotation. In this geometry a vector potential formulation of Maxwell's equations of electromagnetism was solved using the Finite Element Method (FEM) for space discretization. Since solution only takes place in the plane of rotation, only the axial component $A_{z}$ of the vector potential is needed. For time discretization finite differences have been 
used. The induced voltage was calculated by integrating the axial component of the electrical field over all conductors of each phase and then dividing by the area of one conductor. This voltage was then used to calculate the armature winding currents using a coupled circuit model containing the winding and load resistances. The PMs were modeled as regions of constant magnetization, that is

$$
\vec{B}=\mu_{0}\left(\vec{H}+\vec{M}_{P M}\right)
$$

where $\vec{B}$ is magnetic flux density, $\vec{H}$ magnetic field and $\vec{M}_{P M}$ the remanent magnetization of the PM. The non-linearity of the iron was modeled in the simulations by a one-to-one relationship of the kind

$$
\vec{H}=f(|\vec{B}|)
$$

where $f$ is given by interpolation using a lookup table of magnetic flux density versus magnetic field. For the stator steel data for the steel grade was available and used. For the rotor steel, steel grade had not yet been decided and data for a generic soft iron was used. The remainder of the geometry was considered to be free space. This approach is based on the method described by Hannalla in 1981 [12].

Using periodic boundary condition, and the rotational symmetry around the shaft of the machine, the problem was reduced to solving only for a $45^{\circ}$ sector of the geometry. The model was implemented in commercial FEM software COMSOL (www.comsol.com). The simulation resulted in a two dimensional magnetic field in the generator. From this the air gap flux density, induced voltage, armature reaction, and forces on various parts of the generator, could be calculated. Forces on components were calculated using integration of Maxwell's stress tensor over the boundary of the component. To calculate the torque on the rotor Arkkio's method was used instead of ordinary integration of the Maxwell stress tensor for greater accuracy ([13], p. 56).

Results were compared to the in-house generator design program KALK, which has been experimentally verified [2,3]. The comparison was made by comparing the output voltage and fundamental of the air gap flux density waveform, and showed good agreement. Static 3D FEM simulations of the magnetic field in the machine was also made to investigate end effects. These simulations was made both without armature current and with the rated armature current.

For the mechanical design and the mechanical simulations, a commercial CAD software, SolidWorks (www.solidworks.com), was used. Simulations were carried out to check that all parts fitted together, that there is enough space to fasten bolts during assembly, as well as to calculate deformations under expected magnetic forces. Simulations were also run to find the natural modes of vibration of the structure. To speed up simulations the geometry of the parts were simplified and the bolted joints modeled as bonded. Some parts were also removed completely when they were judged to be of low significance to the result of the simulation.

\section{Results}

\subsection{Electromagnetic Design}

Most aspects of the electromagnetic design were determined by mechanical constraints. The optimization that took place was to fit as large PMs as possible into the design to maximize the air gap flux density. 
This was done in a two-step process where an approximate desired size was determined using FEM simulations and then used when looking for standard size magnets to be used in the design.

For ease of manufacture the shape of the pole shoe toward the air gap was chosen to have three planar faces. The middle face is parallel to the tangent of the stator inner perimeter opposite of the pole center, while the two faces on the side taper away from the stator. The width of and angle between the faces were then chosen to make the air gap width approximate $180^{\circ}$ of an inverse sine curve for one pole. This was done to reduce the rotor induced harmonics in the air gap flux density waveform. The inverse sine shape is very close to a shape derived to produce a sinusoidal air gap flux density distribution for a smooth stator under idealized conditions reported by Hauge in 1924 [14].

No rigorous design optimization of the electromagnetic design was carried out, instead effort was put into producing an easy to build design. To make sure the PMs were not in danger of demagnetization under rated operation, the magnetic field in the PMs during operation at rated power was checked.

\subsection{Mechanical Design and Feasibility of Construction}

Once the basic topology of the rotor part of the magnetic circuit had been chosen, consideration was given to how it should be implemented. The rotor topology chosen was a spoke design with tangentially magnetized PMs placed between pole shoes. It was decided that the pole shoes should be fastened with bolts on their inner side. Rough calculations of the forces expected to act upon the pole shoes gave the minimum bolt diameter required, limiting the minimum width of the inward side of the pole. This constraint was then used in the electromagnetic design.

A technical perspective drawing of the rotor design can be seen in Figure 1.

Figure 1. Isometric cut-away view of the ferrite rotor design. Half of the rotor has been removed to make the internal structure more visible. Parts indicated are: (a) fastening flange; (b) L-shaped pole shoe holder; (c) rotor bottom plate; (d) small stiffening ring; (e) top rotor end plate; (f) large stiffening ring; (g) bar for holding the magnet in place; (h) permanent magnet; (i) inner supports; (j) pole shoe; and (k) shaft.

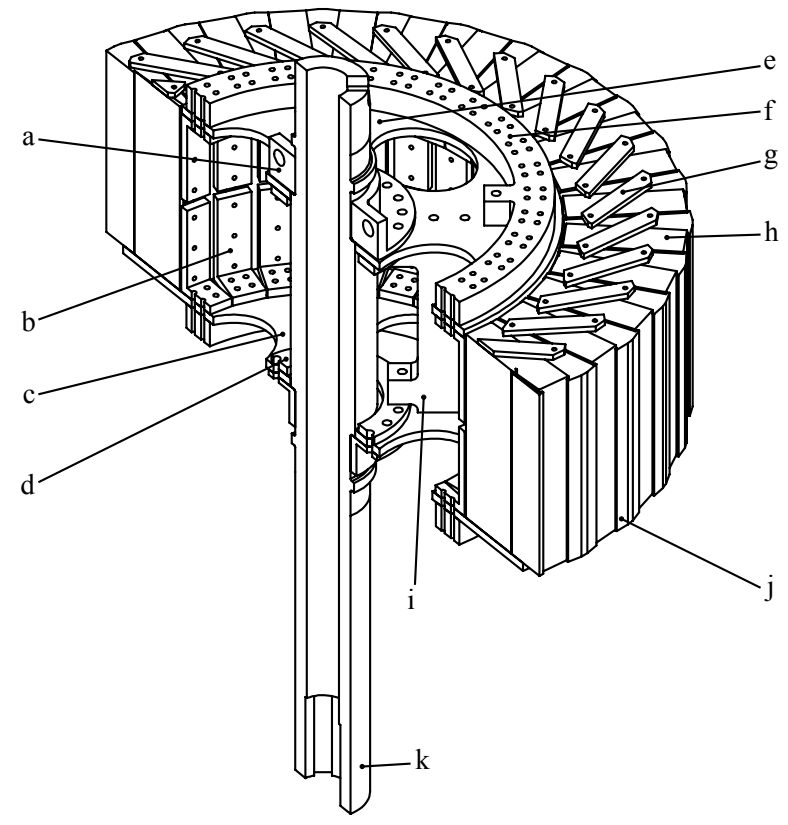


For ease of assembly and structural reasons, laminated poles were opted against, in favor of solid poles. Eddy currents in the solid poles is not expected to pose a problem, since the magnetic flux does not vary significantly in the rotor poles. The pole shoes were mounted on a support structure, consisting of two rotor end plates with plate struts in-between, using a pair of L-shaped holders per pole (in Figure 1c,e,i,b, respectively). The rotor support structure is then fastened to the generator shaft using two flanges (a in Figure 1) that are bolted together. Aluminum was used for most of the support structure, due to lower weight and ease of manufacture, as aluminum is considered to be easier than nonmagnetic steel to machine into required shapes. The lower fatigue resistance of aluminum is not a problem, since long time operation of the research prototype is not expected.

The bolted joints were calculated in a simplified manner with factors of safety in the range of 3 to 7 . The friction between the clamped parts should be sufficient to transfer the shearing forces to avoid shearing the bolt. To ensure this the shearing force times a friction coefficient was added to the tension force to get the required minimum clamping force.

In the final stages of the mechanical design the design was reviewed and plans for how it should be assembled was made, to ensure that all parts could be mounted.

The forces and torques on the pole shoes and rotor as a whole were obtained from the electromagnetic simulations for the three cases: Normal operation, three phase short circuit to ground and two-phase phase-to-phase short circuit. The case with the largest force was then used when computing the stresses. The force imbalance resulting from an off-center rotor was also calculated.

The force on a single pole shoe during normal operation varies between $1400 \mathrm{~N}$ and $1500 \mathrm{~N}$ for the radial component and around $65 \mathrm{~N}$ for the tangential component. Worst case loading, the two phase short circuit, gives the radial component at about $2000 \mathrm{~N}$. Torque on the rotor during normal operation is $930 \mathrm{Nm}$ with a ripple at six times the electrical frequency. During a two-phase short circuit the torque can rise to as much as $8300 \mathrm{Nm}$. A $3 \mathrm{~mm}$ displacement of the rotor from being concentric with the stator creates a force unbalance of $10 \mathrm{kN}$.

The rotor end plates have holes to allow access to the interior of the rotor, which is needed both for assembly and for later experiments with the generator. Slotted spring pins are used to position the pole shoes relative to the bottom rotor end plate. Radial positioning of the bottom rotor end plate relative to the shaft is done with a tight clearance fit. All joints in the design are held together by bolts.

To hold the rotor relative to the stator two composite boards and eight steel bars (not shown in Figure 1) are used. The steel bars are fastened to the stator using dove tail keys. The composite boards are fastened to the bars using bolts and positioned using ledges on the end of the bars. The bearing housings are positioned by a narrow clearance fit with the composite boards and held in place with bolts.

The height of the pole shoes is the same as the height of the stator, $224 \mathrm{~mm}$. The average PM will be slightly higher since a slightly too high magnet had to be chosen to ensure the PM always is the full height of the pole shoe because of tolerances. The size of the magnets in the plane of rotation is $38 \mathrm{~mm}$ by $122.5 \mathrm{~mm}$ with magnetization parallel with the shorter side.

The initial material thickness of the L-shaped holders, $b$ in Figure 1, was chosen as $10 \mathrm{~mm}$ because of available standard extruded aluminum profiles. The thickness of the rotor end plates, $\mathrm{c}$ and e in Figure 1, was chosen as $10 \mathrm{~mm}$. The shaft was chosen to have the same outer diameter $95 \mathrm{~mm}$ outer diameter as the old design. These material thicknesses gave sufficient strength to withstand the expected magnetic 
and inertial loads on the structure during the worst case fault, a two-phase short circuit at rated speed. The strength was also sufficient to keep deformations under acceptable values.

The stiffness against an unbalanced magnetic pull was also investigated. Simulations were made with the force imbalance of $10 \mathrm{kN}$, calculated for a $3 \mathrm{~mm}$ off-center displacement of the rotor, applied to the undeformed generator. To realistically distribute the force on the poles, the unbalanced magnetic pull was superimposed to the attractive force between rotor and stator at nominal air gap. The change in force on each pole from the force at normal air gap was assumed to be proportional to the change in air gap width when the rotor is displaced and directed in the radial direction. The reaction force on the stator was modeled similarly but with a continuous stress distribution rather than discrete forces acting on segments. The magnetic forces are modeled as acting on the surface of the poles and stator. On the poles the surface stress is constant for each pole and applied normal to the air gap face of the pole. On the stator a continuously varying surface stress distribution is used to apply the magnetic force. The magnetic force on the rotor and stator are matched so that there is no net magnetic force. To simplify simulations the stator teeth were removed from the model since it is mainly the yoke of the stator that holds the stator together. Results of the simulations can be seen in Figure 2.

Figure 2. Deformation of the generator under the mechanical loading predicted for a $3 \mathrm{~mm}$ off-center rotor applied to the undeformed geometry. The resultant $10 \mathrm{kN}$ force on the rotor is directed along the red arrow. Some parts of the generator geometry have been hidden to make the relevant parts more visible.

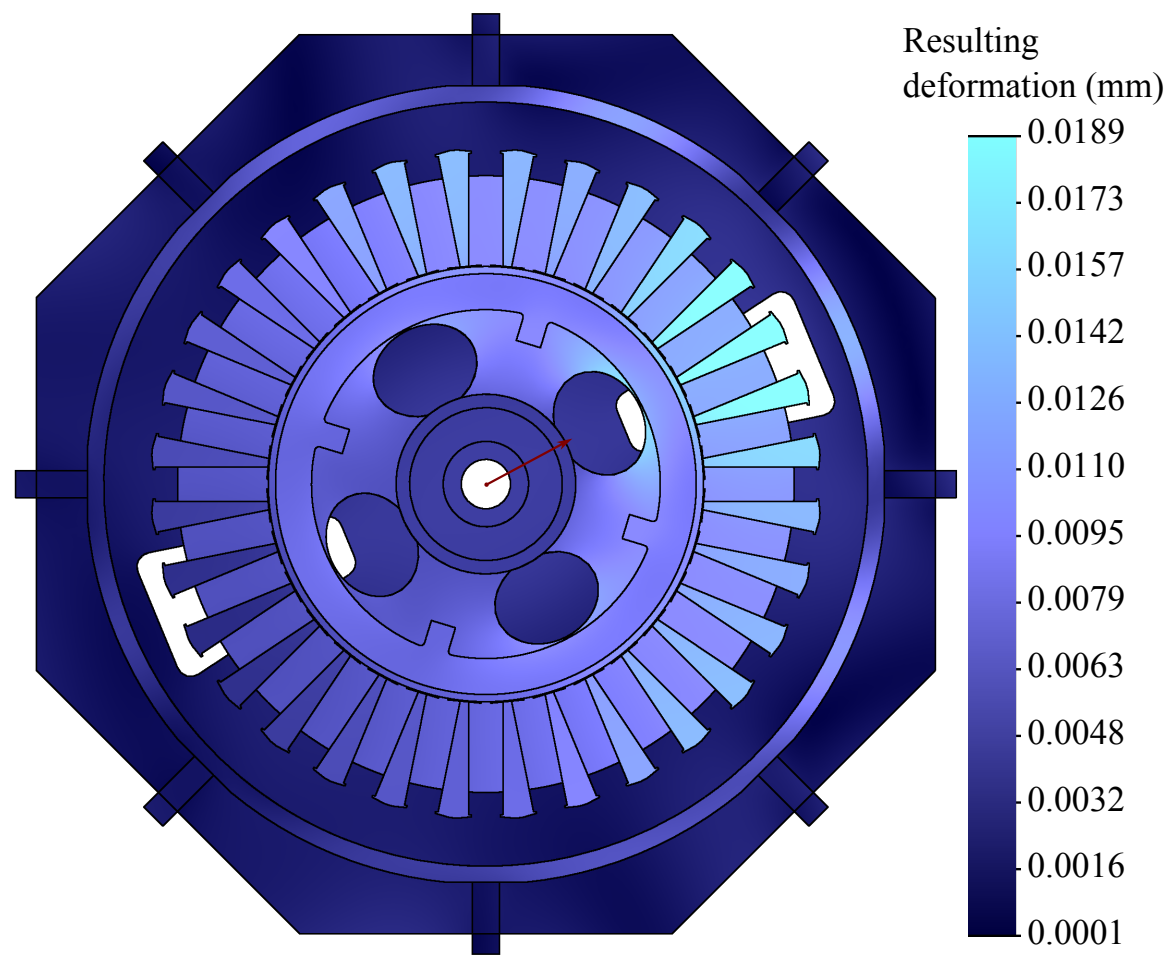

The simulation showed that the unbalance in magnetic pull will only cause displacements smaller by more than one order of magnitude than the displacement required to create it, meaning that the structure is sufficiently stiff. If this was not the case, an off-center displacement or rotor eccentricity would give 
an unbalanced magnetic pull that resulted in a magnetic force that grew faster with deformation than the mechanical force needed to cause the deformation, resulting in the structure collapsing.

Simulations to investigate the natural vibrations of the structure showed modes of vibration at frequencies below the frequency of the torque ripple. The simulations were not expected to give accurate values of the frequencies, due to their simplified nature, but rather to identify modes of vibration that can cause problems. The first mode is shown in Figure 3 and has a frequency of $53 \mathrm{~Hz}$ according to simulations. The structure is held in place by considering four of the lower end of the bars joining the stator and end boards fixed. The stator has been simplified by removing the teeth, the density of the material was then set to keep the mass of the stator constant.

Figure 3. First natural mode of vibration. Deformations are not to scale and exaggerated to show the shape of the mode. The red boxes indicate where the structure is fixed.

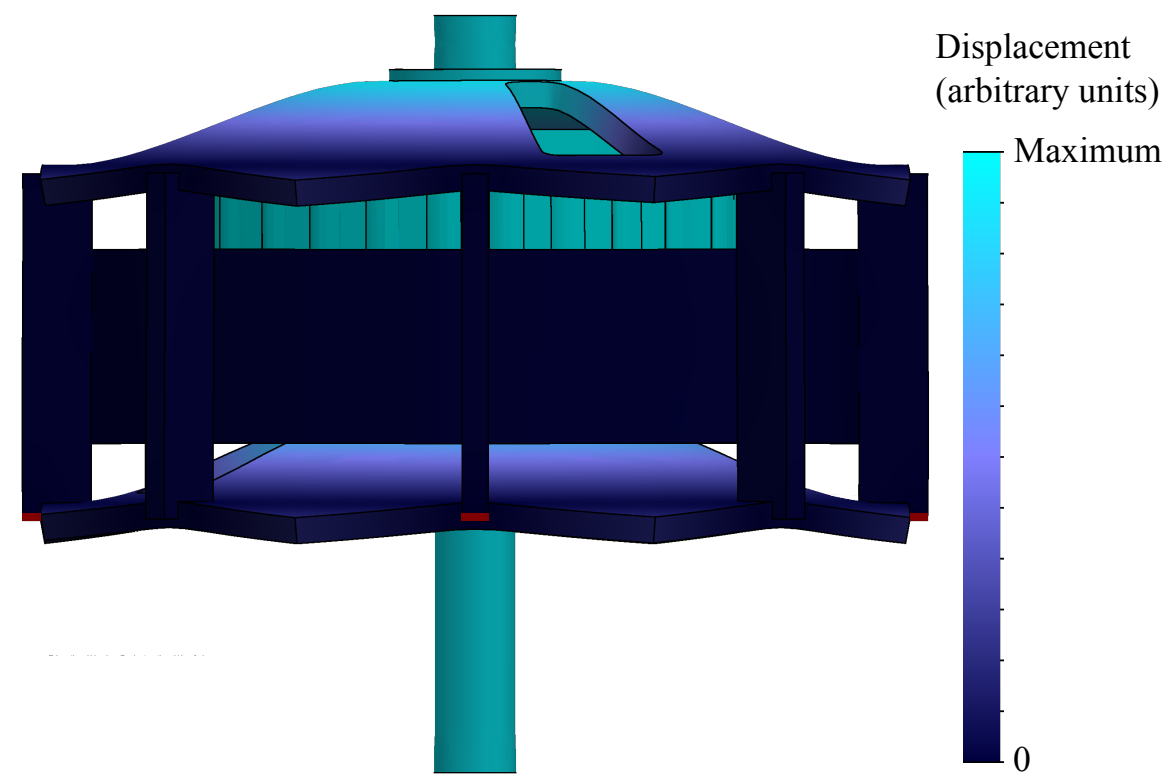

In order to reduce the risk of the discovered modes of vibrations causing problems parts were added to make the structure stiffer. The inner supports, in Figure 1i, were added to counteract the first mode, where the pole shoes and PMs moves up and down relative to the stator by flexing the rotor end boards, in Figure 1c,e, and the composite generator end boards, not shown. The thickness of the generator end boards was also increased to $30 \mathrm{~mm}$, from the $20 \mathrm{~mm}$ used in the old design. The inner supports also stiffen the structure against modes where the rotor oscillates by tilting relative to the shaft. The large stiffening ring, Figure 1f, was added to prevent the rim of the rotor from oscillating in a transversal motion but also positively impacts the tilting modes. To counteract a mode where the rotor oscillates in the plane of rotation by bending the shaft the diameter of segment of the shaft inside the rotor was increased to $110 \mathrm{~mm}$. The inner diameter of the shaft was also decreased from $85 \mathrm{~mm}$ to $55 \mathrm{~mm}$.

In the final stages of the mechanical design the design was reviewed and plans for how it should be assembled was made, to ensure that all parts could be mounted. 


\subsection{Electromagnetic Properties}

The electromagnetic performance of the new design has been predicted through time stepped FEM simulations. A plot showing the magnetic flux density in the generator at load can be seen in Figure 4.

Figure 4. Magnetic flux density and field lines at load, for a $45^{\circ}$ sector of the geometry, obtained through Finite Element Method (FEM) simulations. The axis are position relative to the center of rotation and the color scale is magnetic flux density.

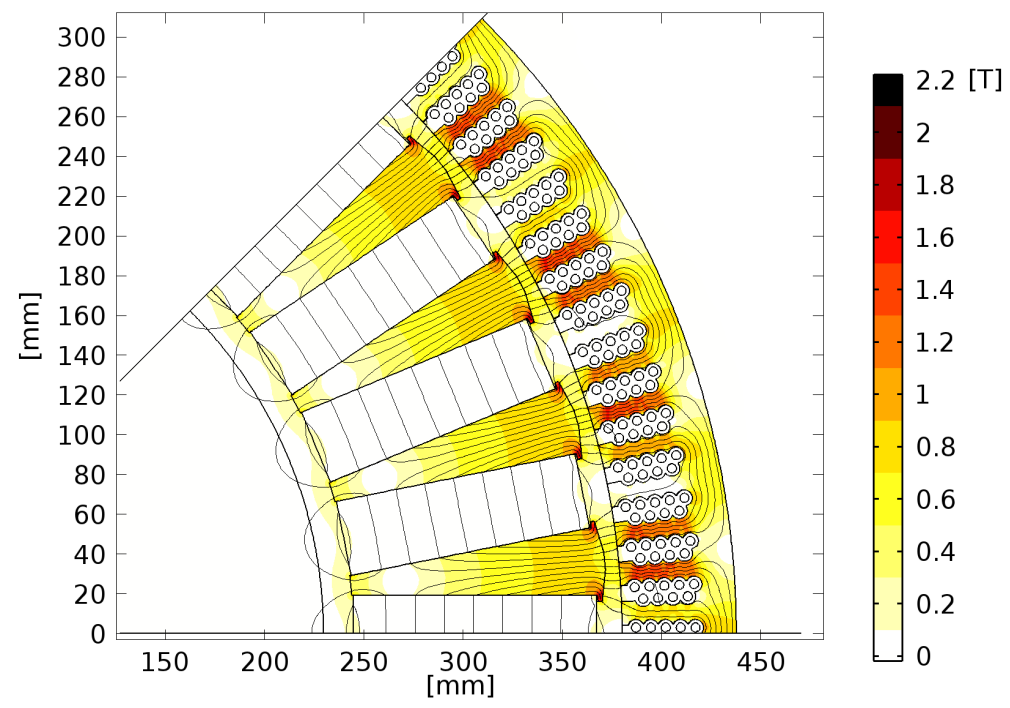

The radial component of the air gap flux density, at no-load, is shown in Figure 5 and the no-load voltage is shown in Figure 6.

Figure 5. The radial component of the air gap flux density waveform and the fundamental of the waveform at no-load, from FEM simulations.

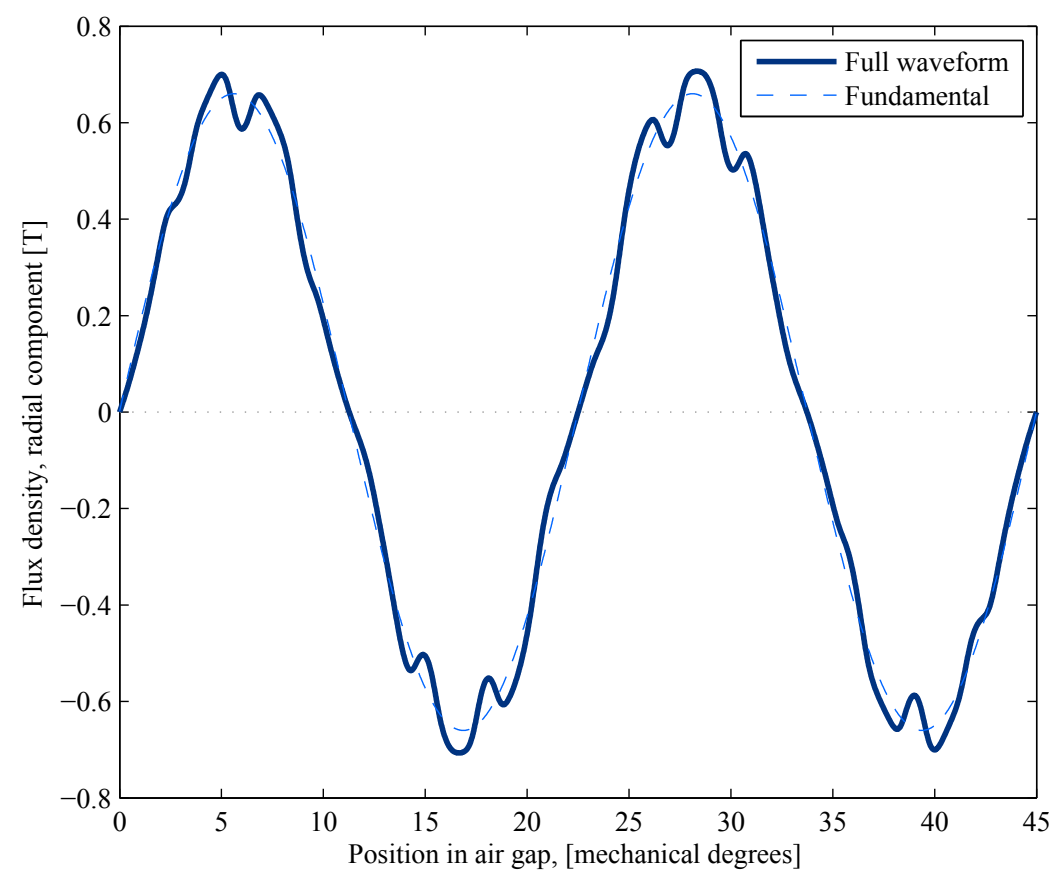


Figure 6. The phase voltage at no-load with the fundamental shown, from FEM simulations.

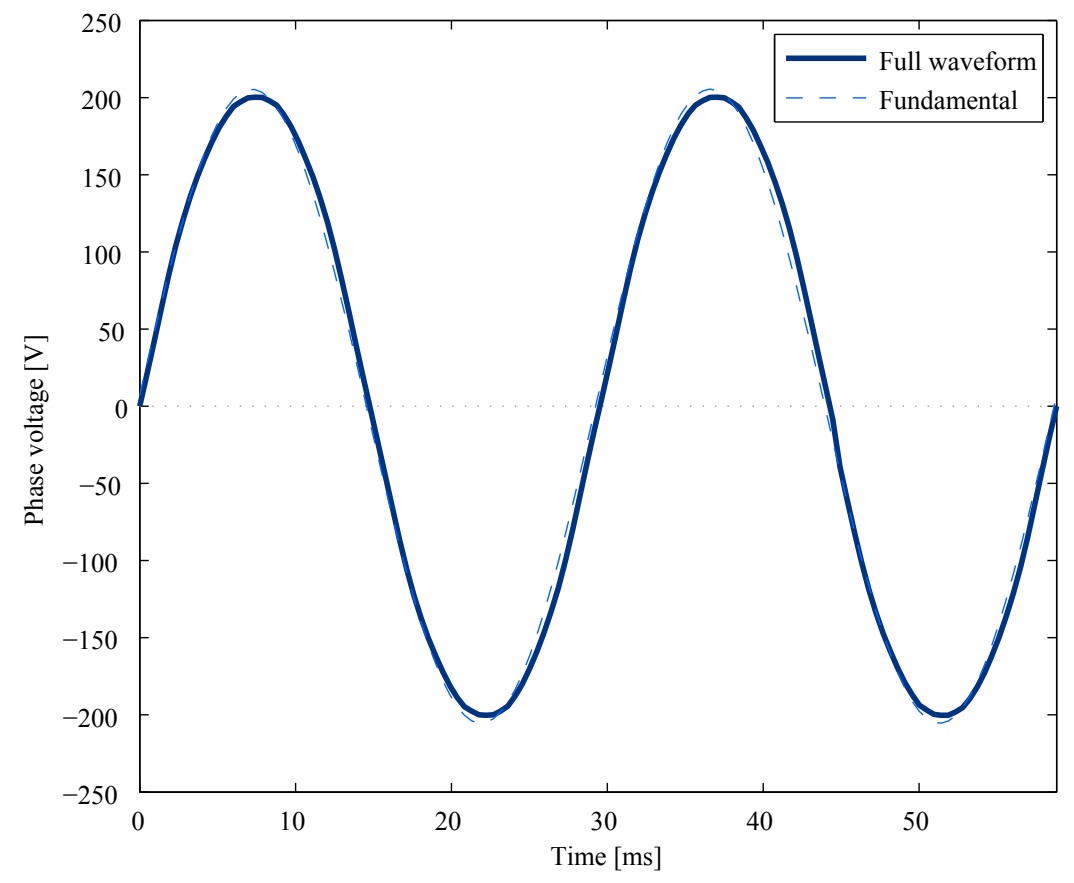

A comparison between the new and original design can be found in Table 2. All data for the new design are from dynamic simulations with the method described in section 4, apart from the iron losses which are taken from stationary simulations with the in-house program KALK. The data for the original design are from dynamic simulations with in-house program KALK apart from the iron losses which are taken from stationary simulations.

Table 2. A comparison of the characteristics of the new design, using ferrite permanent magnets (PMs), and the original design, using neodymium-iron-boron (NdFeB) PMs. Values are at rated load and speed. Data for the new design are from simulations. Data for the original design are from simulations except for weights. Data for the PMs are manufacturers specifications (Sura Magnets AB, (www.suramagnets.se), for the NdFeB PMs and BJA Magnetics, (http://www.bjamagnetics.com), for the ferrite PMs).

\begin{tabular}{lcc}
\hline Quantity & NdFeB (Old) Design & Ferrite (New) Design \\
\hline Rated power [kW] & 12 & 12 \\
Phase voltage, no load (rms) [V] & 172 & 146 \\
Phase voltage (rms) [V] & 167 & 141 \\
Armature current (rms) [A] & 23.9 & 28.5 \\
Armature winding current density (rms) [A/mm $\left.{ }^{2}\right]$ & 1.49 & 1.78 \\
Amplitude of air gap flux density fundamental at no & 0.79 & 0.66 \\
load [T] & & \\
Resistive losses [W] & 275 & 390 \\
Iron losses [W] & 254 & 159 \\
Electromagnetic efficiency [\%] & 95.8 & 95.6 \\
\hline
\end{tabular}


Table 2. Cont.

\begin{tabular}{lcc}
\hline Quantity & NdFeB (Old) Design & Ferrite (New) Design \\
\hline Minimum air gap [mm] & 10 & 7 \\
Mass of rotor [kg] & 130 & 407 \\
Mass of PM [kg] & 41 & 158 \\
Moment of inertia [kg/ $\left.{ }^{2}\right]$ & 16.9 & 34.2 \\
PM material grade & $\mathrm{N} 40$ & $\mathrm{Y} 40$ \\
Remanence of PM [T] & 1.27 & 0.45 \\
Maximum energy product $\left[\mathrm{kJ} / \mathrm{m}^{3}\right]$ & 310 & 47.6 \\
\hline
\end{tabular}

The magnetic flux per pole in the 3D simulations was about $10 \%$ lower than that of the 2D simulations. There was little variation in flux per pole as the armature current was changed from none to rated load current. The results are in line with prior results obtained [8].

\section{Discussion of Results}

The ferrite generator will have a slightly lower output voltage. However, this is acceptable since the generator uses a full converter for grid connection, due to variable speed operation. To maintain the same output power as the original design, the current needs to be increased due to the lower output voltage, increasing the resistive losses in the generator. This can be handled since the original design had quite low current density in the armature windings. The low current density was chosen to give the generator large over-load capability. The decrease in air gap flux density causing the lower voltage will decrease the iron losses. This reduces the decrease in efficiency caused by the increased resistive losses somewhat.

The magnetic flux density plot shown in Figure 4 reveals some leakage flux between poles in the interior of the rotor. No way of eliminating this, without complicating the mechanical structure of the machine, have been found.

One change to the design, that could simplify the manufacturing, would be to stack the poles using thick, about $30 \mathrm{~mm}$, steel plates instead of using solid poles. This would allow the pole shoes to be cut, using laser cutting or similar techniques, and reduce the amount of machining required compared to making the whole pole shoe in a single piece. It would on the other hand make tolerancing and fastener arrangement more cumbersome.

The use of aluminum in the support structure would be questionable in a generator intended for long term operation, since aluminum is sensitive to material fatigue and a generator is subject to cyclic mechanical loads. The machine design presented here is, however, intended for experimental use only and will not be subject to long term operation. For a machine expected to be operated continuously most aluminum parts would have to be replaced by non-magnetic stainless steel, or some other non-magnetic material with better fatigue properties.

The increased weight of the ferrite rotor will add some cost, but also rotating mass. Greater rotating mass makes the generator more stable and easier to control since it filters out fast variations in the applied torque. 
Good agreement between the new simulation method and the experimentally verified simulation tool KALK indicates that the simulations are valid. The comparison did not, however, cover all aspects of the simulations and it is possible that the simulations have errors in some of the untested aspects. The difference between the 3D simulations and the 2D simulations indicate that the $2 \mathrm{D}$ simulations might overestimate the magnetic flux in the machine, if this is the case it will show as a lower than expected output voltage. The design is, however, constrained by the required minimum air gap width, machine length and stator inner diameter in such ways that very little can be done to counteract this in the present case.

The lack of space for the PMs has been a problem during the design process, even though one of the best ferrite magnet material grades on the market has been chosen. This lack of space has caused a lower output voltage than with $\mathrm{NdFeB} \mathrm{PMs} \mathrm{due} \mathrm{to} \mathrm{a} \mathrm{lower} \mathrm{air} \mathrm{gap} \mathrm{flux} \mathrm{density.} \mathrm{Possible} \mathrm{solutions} \mathrm{to}$ this problems would be to either use the available magnetic flux better or increase the magnetic flux. To optimize the stator for the lower flux density, more turns can be added to the armature windings to get higher output voltage. This will, however, give longer stator teeth and possibly reduce the decrease in iron losses caused by lower flux density and might affect the load angle of the machine. The most straightforward way to increase the available flux is to increase the pole pitch to allow larger PMs. Increasing the pole pitch can be done by either increasing the diameter or reducing the number of poles. Given enough axial space, which was not available in the present machine, a rotor with large overhang, as mentioned in the introduction, could also be used to increase the magnetic flux density.

\section{Conclusions}

In this paper, a design process and the resulting design for converting a generator with premagnetized $\mathrm{NdFeB}$ magnets into a novel design with ferrite magnets by replacing the rotor, is presented. The new rotor design can be used with the original stator and has similar properties as the original design. The rated power is achieved at a slightly lower voltage and slightly higher current as a result of lack of space for the ferrite magnets. A larger magnet volume is required to achieve the same air gap flux density and voltage as in the $\mathrm{NdFeB}$ generator, since ferrite magnets are weaker than $\mathrm{NdFeB}$. The resistive losses will increase and the iron losses will decrease resulting in a slightly lower efficiency for the ferrite generator.

The new design will be heavier than the original design but will also have greater rotating mass, reducing the variation in rotational speed due to rapid fluctuations in applied torque. Future work includes building and testing the design.

\section{Acknowledgments}

This work was conducted within the StandUP for Energy strategic research framework. This study was carried out with funding from the Swedish Research Council, Grant Number 2012-4706. 


\section{Author Contributions}

Petter Eklund has written the paper and done all simulations and calculations. Stefan Sjökvist, Sandra Eriksson and Mats Leijon have supervised the work. All four authors have taken part in project planning, design discussions, interpretation of results and manuscript preparation.

\section{Conflicts of Interest}

The authors declare no conflicts of interest.

\section{References}

1. Eriksson, S.; Bernhoff, H. Rotor design for PM generators reflecting the unstable neodymium price. In Proceedings of the IEEE 2012 XXth International Conference on Electrical Machines (ICEM), Marseille, France, 2-5 September 2012; pp. 1419-1423.

2. Eriksson, S.; Solum, A.; Leijon, M.; Bernhoff, H. Simulations and Experiments on a $12 \mathrm{~kW}$ direct driven PM Synchronous Generator. Renew. Energy 2008, 33, 674-681.

3. Eriksson, S.; Bernhoff, H.; Leijon, M. FEM Simulations and Experiments of Different Loading Conditions for a $12 \mathrm{~kW}$ Direct Driven PM Synchronous Generator for Wind Power. Int. J. Emerg. Electr. Power Syst. 2009, 10, 3:1-17:17.

4. Cullity, B.D.; Graham, C.D. Introduction to Magnetic Materials, 2nd ed.; IEEE Press: Piscataway, NJ, USA, 2009.

5. Binns, K.; Kurdali, A. Permanent-magnet a.c. generators. Proc. Inst. Electr. Eng. 1979, 126, 690-696.

6. Muljadi, E.; Butterfield, C.; Wan, Y.H. Axial-flux modular permanent-magnet generator with a toroidal winding for wind-turbine applications. IEEE Trans. Ind. Appl. 1999, 35, 831-836.

7. Jang, S.-M.; Seo, H.-J.; Park, Y.-S.; Park, H.-I.; Choi, J.-Y. Design and electromagnetic field characteristic analysis of $1.5 \mathrm{~kW}$ small scale wind power generator for substitution of Nd-Fe-B to ferrite permanent magnet. IEEE Trans. Magn. 2012, 48, 2933-2936.

8. Dahlgren, M.; Frank, H.; Leijon, M.; Owman, F.; Walfridsson, L. Windformer-Wind Power Goes Large Scale. ABB Rev. 2000, 3, 31-37.

9. Kim, K.C.; Lee, J. The dynamic analysis of a spoke-type permanent magnet generator with large overhang. IEEE Trans. Magn. 2005, 41, 3805-3807.

10. Chen, Z.; Spooner, E. A modular, permanent-magnet generator for variable speed wind turbines. In Proceedings of the Seventh International Conference on Electrical Machines and Drives, Durham, UK, 11-13 September 1995; Volume 412, pp. 453-457.

11. Ekergård, B.; Eriksson, S.; Boström, C.; Leijon, M. Substituting NdFeB with ferrites in linear wave power generator. Renew. Energy 2013, in press.

12. Hannalla, A. Analysis of transient field problems in electrical machines allowing for end leakage and external reactances. IEEE Trans. Magn. 1981, 17, 1240-1243.

13. Arkkio, A. Analysis of Induction Motors Based on the Numerical Solution of the Magnetic Field and Circuit Equations. Ph.D. Thesis, Helsinki University of Technology, Helsinki, Finland, 1987. 
14. Hague, B. The shape of pole-shoe required to produce a sinusoidal distribution of air-gap flux density. J. Inst. Electr. Eng. 1924, 62, 921-929.

(C) 2014 by the authors; licensee MDPI, Basel, Switzerland. This article is an open access article distributed under the terms and conditions of the Creative Commons Attribution license (http://creativecommons.org/licenses/by/3.0/). 